\title{
FRAMELESS STEREOTACTIC NAVIGATION FOR INTRAOPERATIVE LOCALIZATION OF INFECTIOUS INTRACRANIAL ANEURYSM
}

\author{
Felipe Gonçalves de Carvalho', Bruno Loyola Godoy', Marcello Reis', \\ Emerson L. Gasparetto ${ }^{2}$, Eduardo Wajnberg ${ }^{2}$, Jorge Marcondes de Souza
}

Infectious intracranial aneurysms (IIAs) are rare complications of infectious diseases, such as endocarditis and meningitis, caused by the release of bacterial or fungal emboli to the intracranial circulation. The infective emboli can reach the vessel through the endovascular space, as in endocardial infections, or through the extravascular space by contiguous dissemination, in the case of meningitis. Since these emboli tend to be small, they usually reach the distal circulation, causing inflammation in the media and adventitia. This leads to weakening of the vessel wall and aneurysm formation. IIAs account for only 0.7 to $5.4 \%$ of all intracranial aneurysms but are still accountable for high lethality'. Previous studies describe that hemorrhages due to IIA rupture can reach a mortality rate of $80 \%$ and lead to a poor neurologic progno$\mathrm{sis}^{2}$. For this reason, early diagnosis and treatment are essential. Unruptured IIA initial management requires long courses of antibiotic treatment. If the control angiogram shows an enlargement of the lesion, surgical resection is indicated. In the case of ruptured IIAs, the treatment consists of surgical excision ${ }^{3}$.

The localization of IIAs during surgical procedures is usually difficult since they are small, frequently affects distal branches and can be associated with subarachnoid hemorrhage or intracerebral hematomas ${ }^{4}$. Frameless stereotactic navigation has become a valuable operative adjunct device in recent years ${ }^{5}$, but reports of neuronavigation use for intracranial vascular surgery are scarce in the literature. We have encountered a small number of reports confirming the effectiveness of frameless navigation for atypical aneurysms and brain arteriovenous malformations surgeries ${ }^{2,5}$.

We describe the utilization of a frameless stereotactic navigation system for the microsurgical resection of a distal infectious aneurysm.

\section{CASE}

This 34 year-old right handed man was referred to our institution after antibiotic treatment for infectious endocarditis in the two previous weeks. He had a history of rheumatic fever with incomplete treatment in infancy. Transesophageal echocardiography had shown a $3 \mathrm{~cm}$ vegetation in the anterior leaflet of the mitral valve. Blood cultures had identified Streptococcus viridans as the etiologic agent.

Headaches and transient aphasia during the admission motivated a computed tomography (CT) scan that showed a small left frontal hypodense lesion in the middle cerebral artery (MCA) territory distribution, suggesting a previous ischemic event. Cerebral digital angiography was indicated and showed a $2.5 \mathrm{~mm}$ saccular aneurysm in the cortical segment of the MCA. After the administration of culture guided antibiotics, a second control angiography was performed and revealed an increase in aneurysm volume, this time measuring $4.1 \mathrm{~mm}$ (Fig 1A).

The patient was admitted in our institution for surgical treatment and informed consent was obtained. MRI scan with appropriate protocol for neuronavigation was done and demonstrated, on the FLAIR sequence, a small round hypointense lesion in the left superior frontal sulcus. The lesion was localized $1 \mathrm{~cm}$ below to the gyral surface and $2-3 \mathrm{~cm}$ anterior to the left central sulcus. The isquemic signal seen on the previous CT scan was adjacent to the infectious aneurysm (Fig 1B). Using iPlan software (BrainLab, Heimstetten, Germany), preoperative MRI stereotactic images were loaded after facial scanning and the source images were translated into an operative plan (Fig 1C). These images allowed surgical planning and visualization of the infectious aneurysm during surgery, resulting in a simpler procedure with a smaller craniotomy.

The surgical procedure was initiated by a linear scalp incision followed by craniotomy centered at the point indicated by frameless stereotaxy planning. After dural opening a small arachnoid dissection revealed the aneurysm at the bottom of the precentral sulcus. It was isolated and successfully resected (Fig 2).

\section{NEURONAVEGAÇÃO ESTEREOTÁXICA PARA LOCALIZAÇÃO INTRAOPERATÓRIA DE ANEURISMA INFECCIOSO INTRACRANIANO}

Hospital Universitário Clementino Fraga Filho, Rio de Janeiro RJ, Brazil: 'Department of Neurosurgery; ${ }^{2}$ Department of Radiology.

Received 27 April 2009, received in final form 14 July 2009. Accepted 6 August 2009.

Dr. Jorge Marcondes de Souza - Department of Neurosurgery / Hospital Universitário Clementino Fraga Fillho - Av. Rodolpho Rocco 250 - 21941-902 Rio de Janeiro RJ - Brasil.E-mail address: jmarcondes@hucff.ufrj.br 

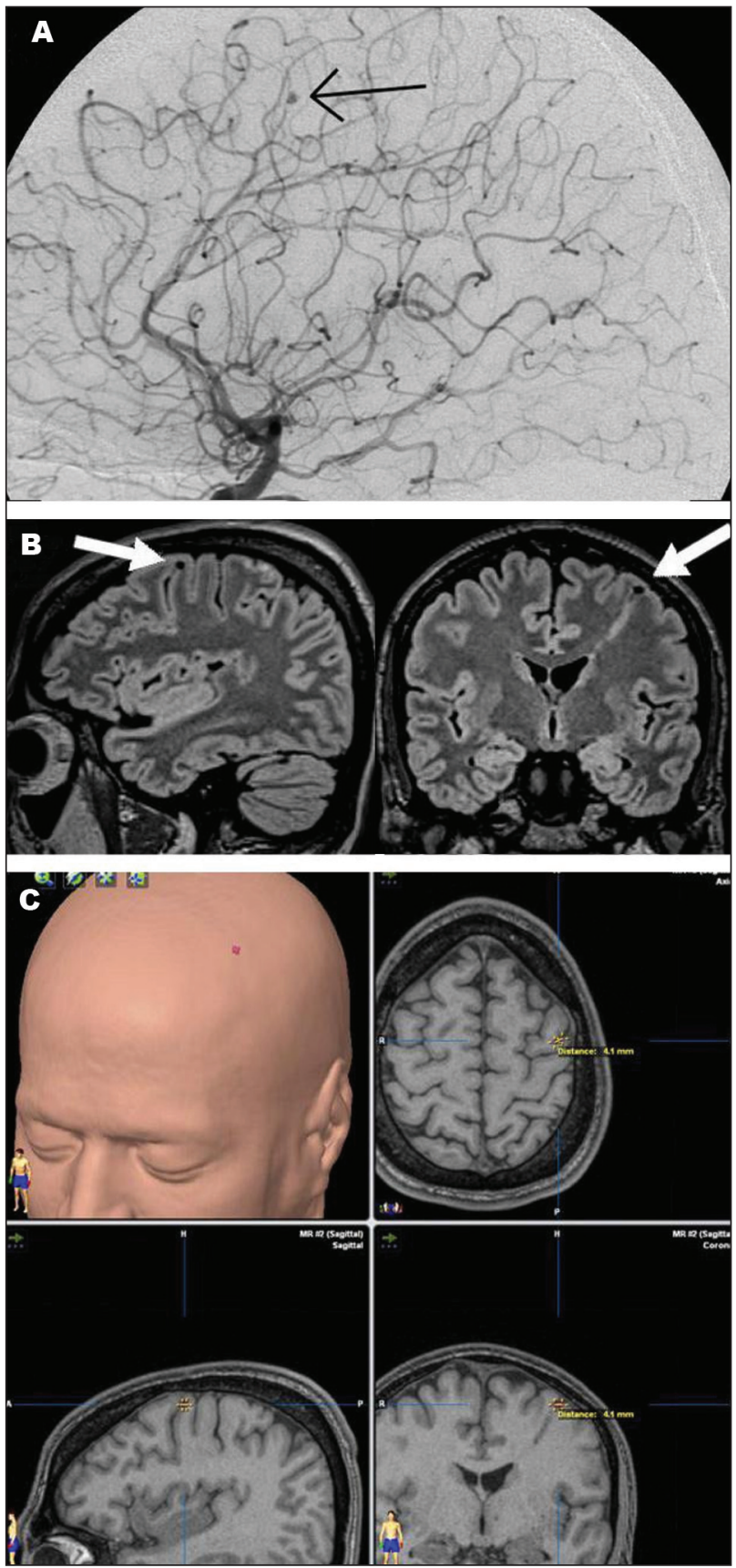

Fig 1. [A] Lateral carotid digital subtraction angiogram showing a saccular aneurysm (arrow) arising from a cortical branch of the left MCA. [B] Sagital and coronal MRI T7WI based images showing a small round hypointense lesion measuring $4.1 \mathrm{~mm}$ with signal void (arrows) located cortico-subcortically near the left precentral sulcus. [C] Three-dimensional reconstructed images showing how BrainLab Navigation System was used for intraoperative localization of the aneurysm with preoperative MRI as a reference.

The patient recovered well and was sent neurologically intact to the referring hospital.

\section{DISCUSSION}

Neurologic complications of endocarditis are common, occurring in 20 to $40 \%$ of patients ${ }^{6}$. However, infec-

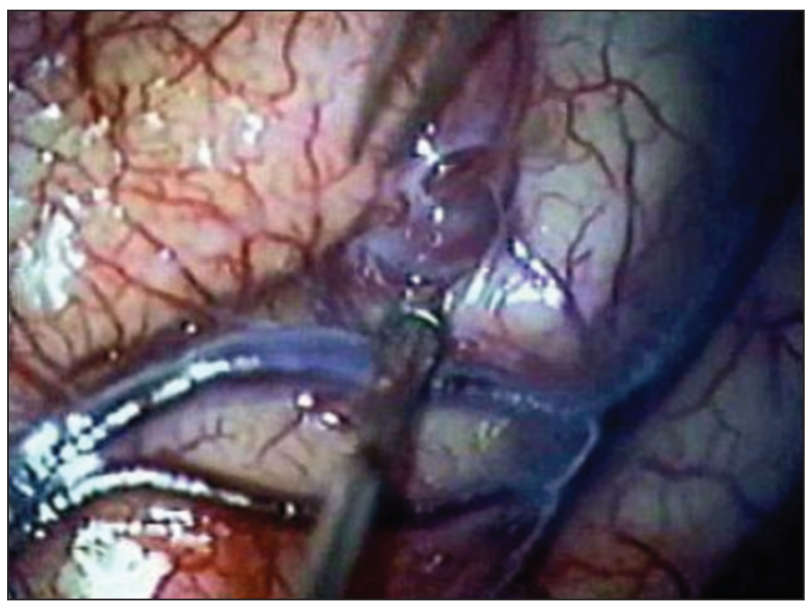

Fig 2. Intraoperative image demonstrates arachnoid dissection and the aneurysm arising at the precentral sulcus from a distal MCA branch.

tious aneurysms are infrequent, $70 \%$ of them being located on the MCA or its distal branches ${ }^{5,7}$. Conservative management with prolonged course of antibiotics and followup angiogram is the initial treatment. Enlargement of the aneurysm indicates progressive wall distension, increasing the risk of rupture. Therefore, surgical treatment should be considered since aneurysm rupture is associated with an $80 \%$ morbidity and mortality ${ }^{1}$. Failure to reduce aneurysm size after the planned course of antibiotic therapy should indicate surgical intervention ${ }^{3}$. In selected cases, an alternative option would be aneurysm occlusion by endovascular approach ${ }^{8}$.

The exact timing for surgery remains a controversial topic. The initial approach should always include antibiotic treatment. This may facilitate surgical resection of IIAs since antibiotics can not only treat the infection but also help aneurysm maturation from a friable acute lesion to a more fibrotic, subacute or chronic lesion. However, clinical management should not delay surgery when an intervention is indicated'.

Origitano et al., among other authors, describe that frameless stereotactic navigation should be considered when the aneurysm is small, distal or at unusual locations, such as in the case of infectious aneurysms ${ }^{8-10}$. In this case report, we have demonstrated the importance of frameless stereotactic MRI-based navigation in the treatment of infectious aneurysms. This technique increases aneurysm localization accuracy, reduces the time for dissection and diminishes the risk of vascular and parenchymal iatrogenic damage.

In summary, we have not found previous descriptions of frameless stereotactic navigation use for infectious aneurysms resection. Difficulties in dealing with IIAs suggest that frameless navigation systems should be considered a useful tool in this scenario. 
ACKNOWLEDGEMENTS - We would like to thank Dr. Adriana Carvalho for revising the manuscript.

\section{REFERENCES}

1. Nakahara I, Taha MM, Higashi T, et al. Different modalities of treatment of intracranial mycotic aneurysms: report of 4 cases. Surg Neurol 2006;66:405-409.

2. Peters PJ, Harrison T, Lennox JL. A dangerous dilemma: management of infectious intracranial aneurysms complicating endocarditis. Lancet Infect Dis 2006;6:742-748.

3. Origitano TC, Anderson DE. CT angiographic-guided frameless stereotactic-assisted clipping of a distal posterior inferior cerebellar artery aneurysm: technical case report. Surg Neurol 1996;46:450-453.

4. Dashti R, Hernesniemi J, Niemelä M, et al. Microneurosurgical management of distal middle cerebral artery aneurysms. Surg Neurol 2007;67:553-563.
5. Carlson JD, Liu JK, Dogan A, Sincoff E, Anderson GJ, Delashaw JB Jr. Use of frameless stereotactic computed tomography venography for intraoperative localization of dural arterial venous fistulas: case report. Surg Neurol 2008;70:521-525.

6. Kannoth S, Thomas SV, Nair S, Sarma PS. Proposed diagnostic criteria for intracranial infectious aneurysms. J Neurol Neurosurg Psychiatry 2008;79:943-946.

7. Pirotte B, Wikler D, David P, et al. Magnetic resonance angiography image guidance for the microsurgical clipping of intracranial aneurysms: a report of two cases. Neurol Res 2004;26:429-434.

8. Nussbaum ES, Madison MT, Goddard JK, Lassig JP, Nussbaum LA. Peripheral intracranial aneurysms: management challenges in 60 consecutive cases. J Neurosurg 2009;110:7-13.

9. Lee SH, Bang JS. Distal middle cerebral artery M4 aneurysm surgery using navigation-CT angiography. J Korean Neurosurg Soc 2007;42:478-480.

10. Phuong LK, Link M, Wijdicks E. Management of intracranial infectious aneurysms: a series of 16 cases Neurosurgery 2002;51:1145-1151. 